\title{
Electronic Detection of DNA Adsorption and Hybridization
}

\author{
Ulrich Bockelmann \\ Laboratoire de Nanobiophysique \\ UMR Gulliver CNRS-ESPCI 7083 \\ 10 rue Vauquelin, 75005 Paris (France) \\ ulrich.bockelmann@espci.fr
}

\begin{abstract}
We consider a transistor based approach for DNA detection. Immobilization of DNA at different positions of poly(l-lysine) coated field effect transistor arrays is achieved by deposition with a microspotting device or specific hybridization between complementary oligonucleotide sequences. The current voltage characteristics of the transistors are measured with the sample surface immersed in aqueous solution. The chapter provides a brief overview of our experimental technique and of its applications to the detection of DNA adsorption and hybridization.
\end{abstract}

\section{Introduction}

The large majority of nucleic acid analysis techniques presently rely on detection schemes which involve fluorescence, radioactivity or enzyme based labelling [1]. In many cases the labelling steps are expensive, time-consuming and error-prone and the corresponding equipment is expensive. These disadventages are major motivations for research on alternative detection methods [2-4].

After early work by P. Bergveld [5], several papers addressed the detection of biomolecules binding on field effect transistor (FET) structures [6-8]. DNA is a strong negatively charged polyelectrolyte in aqueous solutions. Capacitive impedance and FET detection of DNA have been achieved over the last years $[9-14]$.

We use a differential technique based on dc measurements with arrays of integrated FET devices $[12,15,16]$. Our approach allows for efficient miniaturisation and parallelisation through on-chip integration of the detection and readout circuits [17]. It involves adsorption of the biomolecules to part of the array (achieved by local deposition of sub-nanoliter volumes of solutions with a microspotting device) and measurement of the transistors (with the surface of the microchip covered by an aqueous solution).

The chapter is organized as follows. The experimental techniques are presented in Sect. 2. Electronic detection of DNA immobilized by direct adsorption on poly(L-lysine) coated FET arrays is considered in Sect. 3. Sect. 4 is devoted to the detection of specific hybridization between DNA oligonucleotides. Salt effects are briefly considered in Sect. 5. Sect. 6 contains the concluding part of the chapter.

Please use the following format when citing this chapter:

Bockelmann, U., 2007, in IFIP International Federation for Information Processing, Volume 249, VLSI-SoC: Research Trends in VLSI and Systems on Chip, eds. De Micheli, G., Mir, S., Reis, R., (Boston: Springer), pp. 55-67 


\section{Experimental techniques}

\subsection{Fabrication of the FET arrays}

Arrays of silicon p-channel field effect transistors have been used in this work. The samples were fabricated in the clean room facility of the Max Planck Institut für Biochemie, Martinsried, Germany. The process is based on standard silicon microtechnology and is described in $[18,19]$. The active areas of the individual transistors carry a $10 \mathrm{~nm}$ thick $\mathrm{SiO}_{2}$ oxide without metal gate. Typically, 96 FETs are linearly arranged with a period of $20 \mu \mathrm{m}$, the active surface area of an individual FET amounts to 36 or $100 \mu \mathrm{m}^{2}$. The drain of each transistor is individually connected, while the whole array shares a common source contact. On the silicon chip these connexions are realized by boron $\mathrm{p}^{+}$implantation. The chips are mounted on ceramics sockets, wire bonded and finally a plastic well is glued on the surface to protect the wiring during the subsequent manipulations where the surface is incubated with liquids.

\subsection{Surface preparation}

Prior to DNA immobilization, we perform the following global treatment of the $\mathrm{SiO}_{2}$ surface. Incubation in sulfochromic acid, rinsing with $\mathrm{H}_{2} \mathrm{O}$, incubation in a $\mathrm{NaOH}$ /ethanol solution, $\mathrm{H}_{2} \mathrm{O}$ rinsing and drying with air. Afterwards, the FET array is incubated in a poly(L-lysine) dilution, again followed by $\mathrm{H}_{2} \mathrm{O}$ rinsing and drying with air. Immobilization of DNA by a poly(L-lysine) layer is widely used in the field of DNA microarrays, although the resulting attachment is less stable than the one obtained by covalent immobilization strategies. DNA immobilization with poly(L-lysine) is relatively simple and allows us to use a FET array several times. In fact, we can clean the $\mathrm{SiO}_{2}$ surface after use and repeat DNA immobilization and detection.

\subsection{Local deposition of DNA}

A piezo spotting device equiped with a custom microscopic imaging system is used to deposite DNA solutions on the FET array, as shown in Figure 1. Typically spot diameters of $50-200 \mu \mathrm{m}$ are used, corresponding to volumina in the 0.1-1 nl range.

\subsection{Electronic measurements}

For the electronic measurements the surface of the chip is immersed in aqueous electrolyte solution, together with a reference electrode. We measure the drain current $I_{D}$ of each transistor as a function of a dc voltage $U_{S D}$ applied between source and drain and a dc voltage $U_{S E}$ applied between source and the reference electrode. These 2D characteristics are recorded at room temperature. An analog amplifier circuit is used to bias the FETs and to measure the drain current $I_{D}$, as shown in Figure 2. The individual FETs of the array are multiplexed by a 


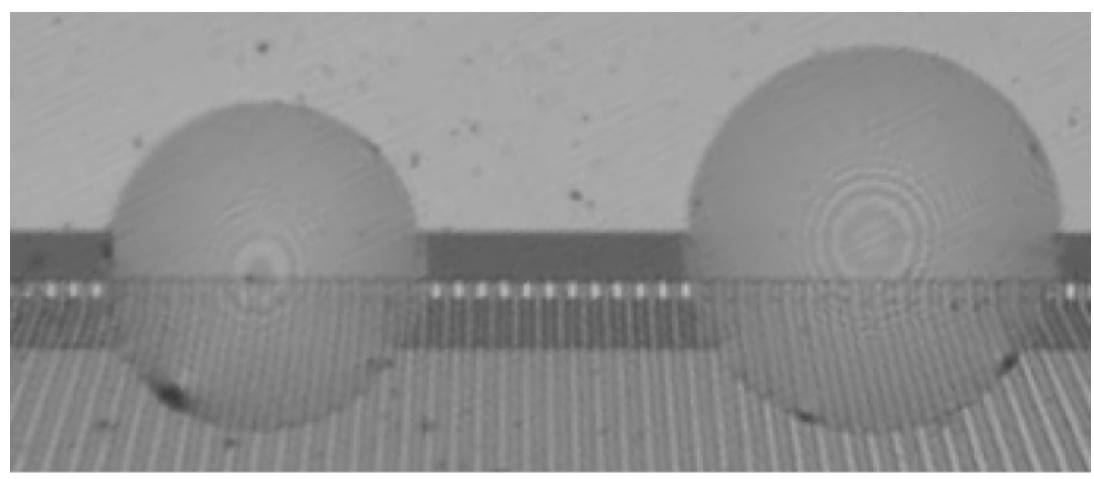

Fig. 1. Microscopic image of a transistor array with two local deposits. The spots have diameters of about $200 \mu \mathrm{m}$ and each one covers about 10 FETs of the linear array. The active regions of the individual transistors appear as white squares in the image. The gray lines in the bottom part are the individual drain connexions. The homogeneous region in the upper part is the common source contact.

switch unit, the latter being computer controlled by a digital I/O board. Two dc voltages $\tilde{U}_{S E}, \tilde{U}_{S D}$ are generated by 16 bit $\mathrm{D} / \mathrm{A}$ converters of a multifunction $\mathrm{I} / \mathrm{O}$ board and are lowpass filtered in the input stage of the amplifier. The drain current is converted to a voltage that is measured by a 16 bit A/D converter of the I/O board. The acquisition of the bidimensional characteristics $I_{D}\left(\tilde{U}_{S D}, \tilde{U}_{S}\right)$ for all FETs is computer controlled.

Dependending on the design of the FET array device the implanted on-chip connexions to the individual drains and the common source may lead to nonnegligible serial resistances. In this case, we consider the lateral geometry of the connexions and derive the serial resistances of the drain lead $R_{D}$ and the source lead $R_{S}$ for each FET. The transformation

$$
\begin{aligned}
U_{S E} & =\tilde{U}_{S E}-R_{S} I_{D} \\
U_{S D} & =\tilde{U}_{S D}-\left(R_{D}+R_{S}\right) I_{D}
\end{aligned}
$$

finally gives the intrinsic characteristics $I_{D}\left(U_{S D}, U_{S E}\right)$. To study shifts in $U_{S E}$ we convert the $I_{D}\left(U_{S D}, U_{S E}\right)$ characteristics, to $U_{S E}\left(I_{D}, U_{S D}\right)$ by numerical interpolation.

\subsection{Microfluorescence}

For comparison with the electronic signals we use microscopic fluorescence measurements. The corresponding optical setup is schematically presented in Figure 3. The beam of a red $(632.8 \mathrm{~nm})$ HeNe $\mathrm{cw}$ laser or a green $(532 \mathrm{~nm})$ solid state $\mathrm{cw}$ laser is expanded, introduced in the illumination path of an upright microscope and focused with a microscope objective to a spot of adjustable diameter (0.5-10 $\mu \mathrm{m})$. Fluorescence is collected by the same objective and passes two 


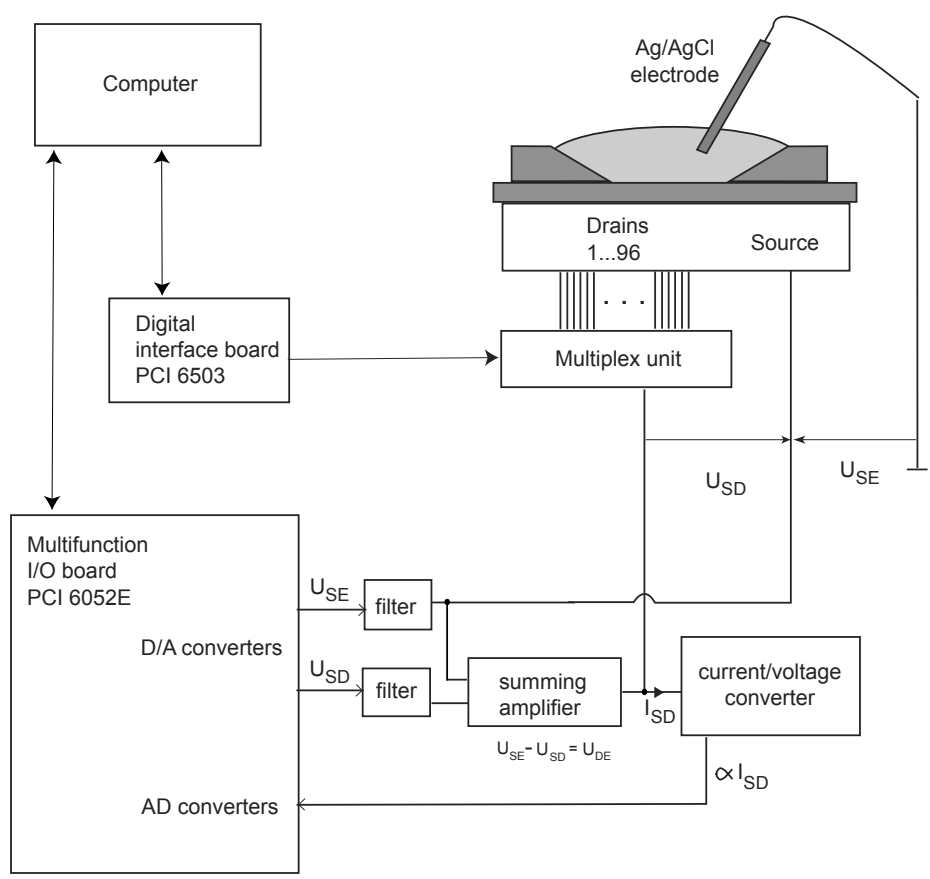

Fig. 2. Recording setup used for the differential detection of biopolymers. The FET array contains one drain connection for each of the 96 transistors and one common source.

crossed piezo-adjustable slits, positioned in an image plane of the sample. The two-slit arrangement defines a rectangular detection region and allows us to optimise this region with respect to the size of the excitation spot. Excitation light is blocked by an emission filter and the fluorescence signal is measured with a cooled photomultiplier tube. A motorized xy translation stage with integrated position sensors operating in a feedback loop is used to scan the sample laterally.

\section{Detecting DNA adsorption}

A typical electronic measurement of DNA adsorption is presented in Figure 4. One deposit of pure water (left) and four deposits of an oligonucleotide solution (right) are done on the surface of a poly(L-lysine) coated array. The horizontal axis indicates the index of the different transistors. The 96 individual transistors of this array are arranged along a line of about $2 \mathrm{~mm}$ in length. Each transistor has an active surface of $100 \mu \mathrm{m}^{2}$. In this experiment, spots of about $200 \mu \mathrm{m}$ in diameter were deposited. The difference in $U_{S E}$ between a first measurement done prior to the deposition and a second measurement done directly afterwards 


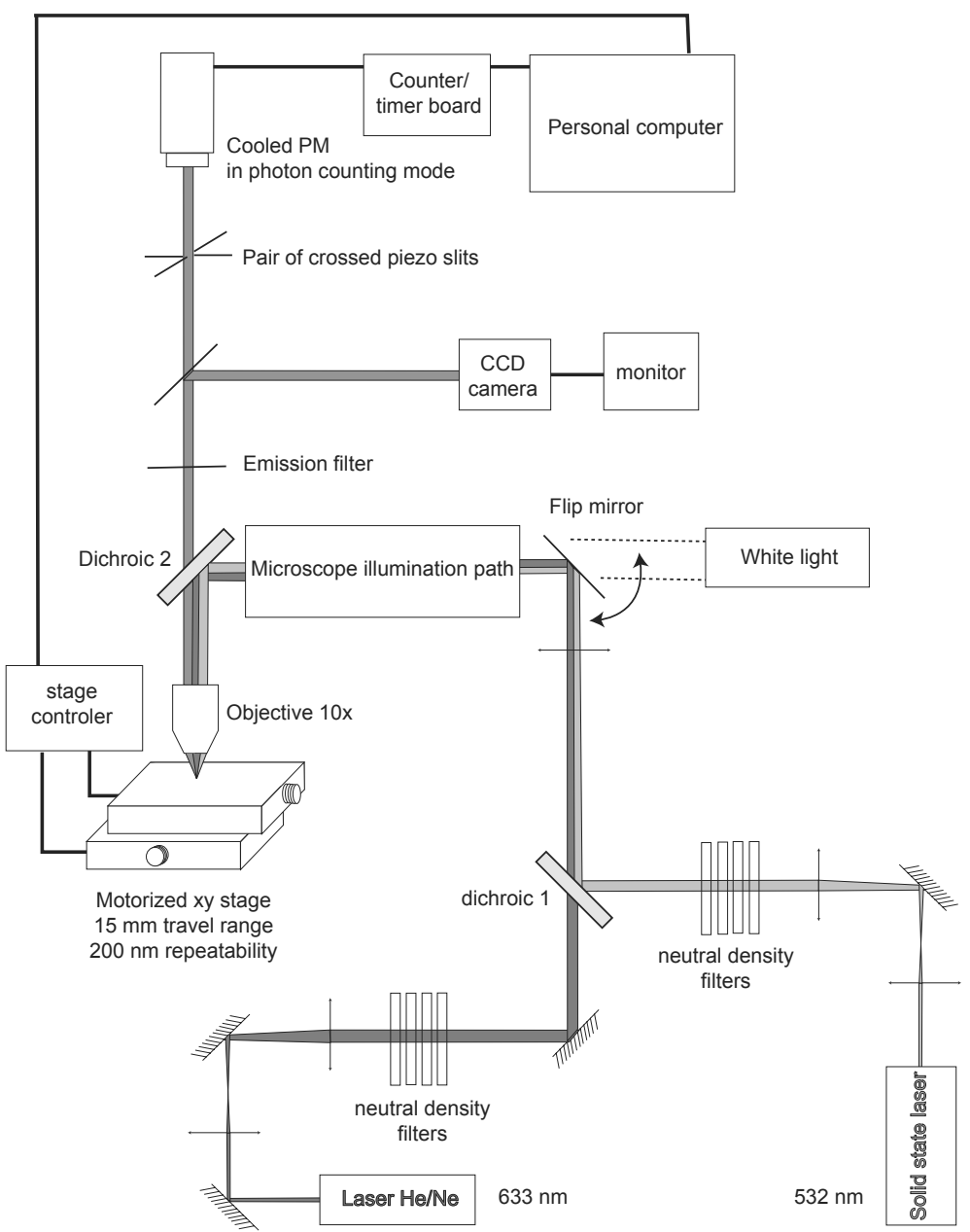

Fig. 3. Dual color fluorescence setup for imaging the transistor array surfaces. 
is presented. The adsorption of DNA gives rise to negative shifts $\Delta U_{S E}$, while for the transistors below the reference spot no significant shift relative to the baseline is measured.

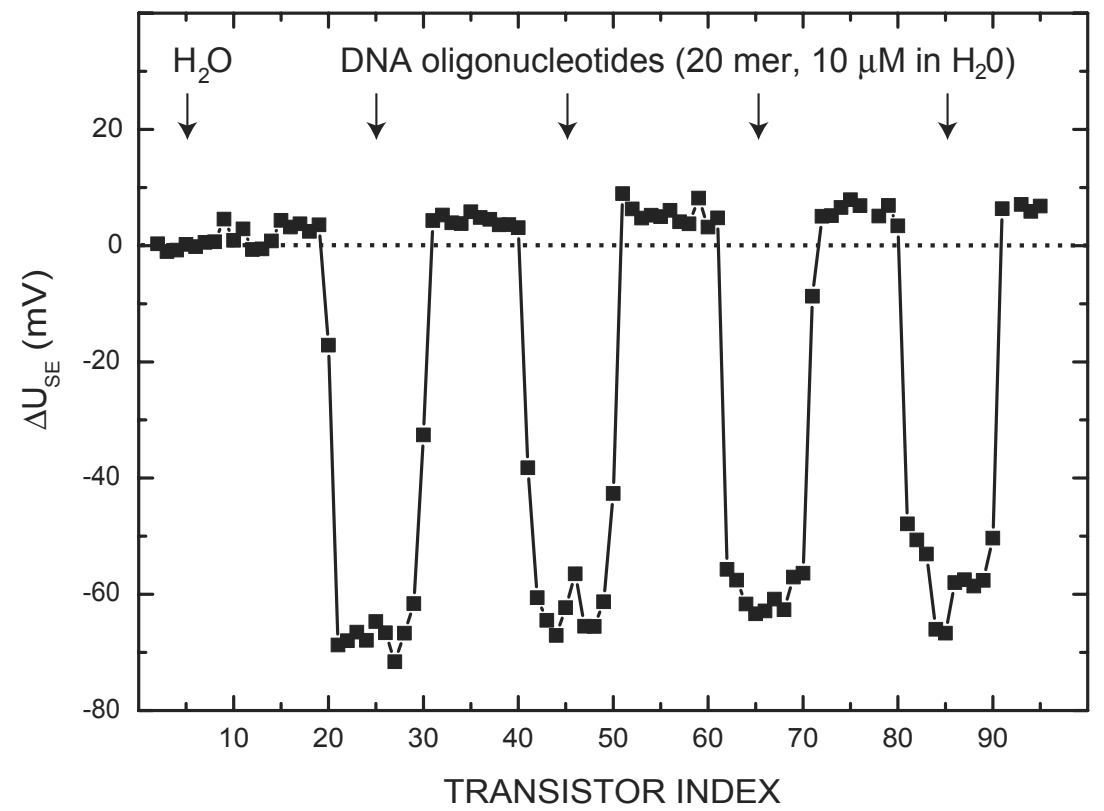

Fig. 4. Electronic detection of DNA adsorbed on a poly(L-lysine) coated array of 96 FETs linearly arranged with a period of $20 \mu \mathrm{m}$. Four local deposits of a DNA oligonucleotide solution are performed and $\mathrm{H}_{2} \mathrm{O}$ spot is added for comparison. The positions of these spots are indicated by arrows. Each data point correspond to one transistor of the array (bottom axis). The quantity $\Delta U_{S E}$ gives the shift of the current/voltage characteristics at a given $\left(I_{D}, U_{S D}\right)$ working point, observed between two measurements. The first measurement is done prior to the deposition, the second one immediately afterwards, in both cases the sample surface was covered by an aqueous electrolyte solution $\left(0.1 \mathrm{mM} \mathrm{KNO}_{3}\right)$. The DNA spots induced negative shifts $\Delta U_{S E}$ of about 60 $\mathrm{mV}$, while no corresponding signal is observed on the reference spot.

In an earlier publication [12], we presented a comprehensive investigation of the electronic detection of poly(L-lysine) and DNA. Adsorption of DNA on poly(L-lysine) coated $\mathrm{SiO}_{2}$ induces negative shifts in $U_{S E}$, while adsorption of poly(L-lysine) on $\mathrm{SiO}_{2}$ leads to positive shifts. This can be attributed to the fact that we use p-channel FET arrays and that DNA carries negative charge in aqueous solution around $\mathrm{pH} 7$, opposite to the positively charged poly(L-lysine). The electronic signals induced by adsorption of these charged polymers were studied as a function of electrolyte salt and polymer concentrations and an analytical model which accounts for screening of the adsorbed charge by mobile ions was 
developed. The microfluorescence setup described above was used in combination with $\mathrm{Cy} 3$ and/or Cy5 modified oligonucleotides to quantify the local DNA adsorption. Non-modified, Cy3-modified and Cy5-modified oligonucleotides are electronically detected without noticable differences. It is also possible to detect the adsorption of double stranded DNA. This was shown with double stranded molecules synthesized in-vitro by PCR (polymerase chain reaction).

In another study, we showed that the FET based measurement is compatible with enzymatic technology and complex DNA samples [15]. We combined the electronic detection with an allele-specific polymerase chain reaction and thus detected a single-basepair mutation in genomic DNA. The approach was applied to test human DNA for the 35 delG mutation, a frequent mutation related to prelingual nonsyndromic deafness.

\section{Detecting DNA hybridization}

Hybridization involves the specific interaction between complementary base sequences and the transition from two single stranded to one double stranded molecule. In general, the single stranded molecule carries a different effective charge than the double stranded one [20,21]. Therefore hybridization between a surface bound probe molecule and a target molecule from solution can change the electrostatic potential of the $\mathrm{SiO}_{2}$ /electrolyte interface and it is conceivable to electronically detect this specific interaction with our FET arrays. Specific recognition of target sequences by hybridization to surface bound probe DNA is the basis of microarray based DNA analysis, a rapidly developping technology with broad applications in research and diagnostics.
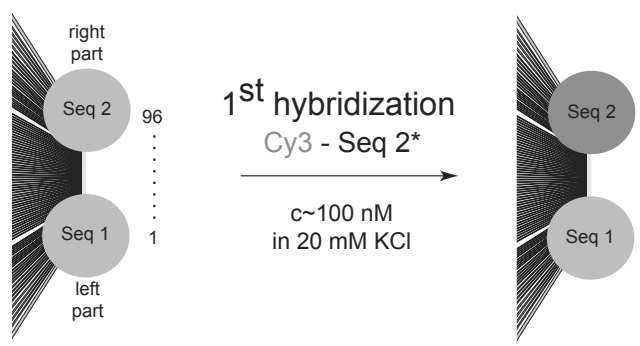

$2^{\text {nd }}$ hybridization Cy5 - Seq $1^{*}$

$\mathrm{c} \sim 100 \mathrm{nM}$

in $20 \mathrm{mM} \mathrm{KCl}$

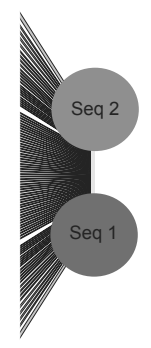

Fig. 5. Detection of hybridization. Oligonucleotide probes with two different base sequences are spotted to the left (transistors 1-31) and right (transistors 54-96) parts of an array of 96 transistors. Each transistor exhibits an active surface of $100 \mu \mathrm{m}^{2}$. The first hybridization is done with $\mathrm{Cy} 3$ labeled target oligonucleotides, matching the probes on the right part of the array. Afterwards, the second hybridization is done with Cy5 labeled targets matching the probes on the left part of the array. 


\section{Electronics}

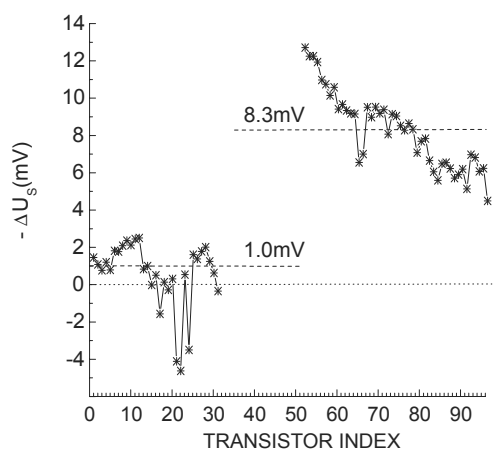

\section{Fluorescence}

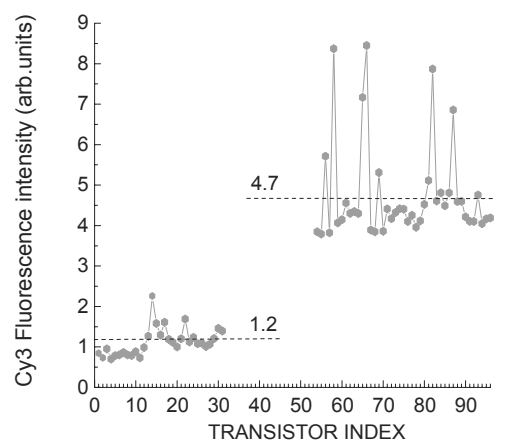

Fig. 6. Detection of the first hybridization. Both electronic (left, $-\Delta U_{S}$ ) and fluorescence (right) signals are higher on the right part of the array, where specific hybridization is expected.

\section{Electronics}

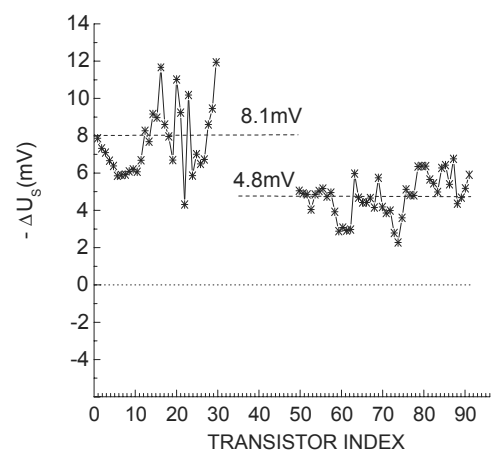

Fluorescence

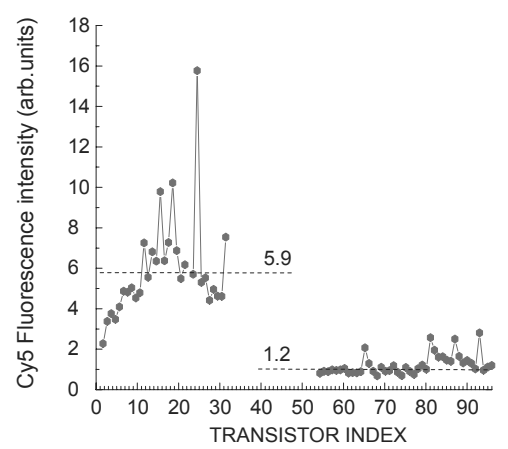

Fig. 7. Detection of the second hybridization. This time electronic and fluorescence signals are higher at the left, as expected. 
Figures 5, 6 and 7 illustrate an experiment showing electronic and fluorescence detection of hybridization between DNA oligonucleotides. After initial cleaning we coated the surface of our planar device with poly(L-lysine). Using a micropipette we then deposited two different oligonucleotide solutions $(0.3$ $\mu \mathrm{l}$ of $1 \mu \mathrm{M}$ solutions of 20 mer oligonucleotides in $20 \mathrm{mM} \mathrm{KCl}$ ) to the left and right parts of the FET array (transistors 1-31 and 54-96, respectively). Incubation was done for 15 min at room temperature, before $\mathrm{H}_{2} \mathrm{O}$ rinsing and drying with air.

For hybridization, the sample is incubated for $5 \mathrm{~min}$ at room temperature in $20 \mathrm{mM} \mathrm{KCl}$ buffer containing target oligonucleotides to a concentration of $100 \mathrm{nM}$. The hybridization is stopped by rinsing with fresh $20 \mathrm{mM} \mathrm{KCl}$ buffer (without target DNA and using a micropipette). The left part of figure 6 shows the difference $\Delta U_{S}$ between two electronic measurements at $[\mathrm{KCl}]=20 \mathrm{mM}$, done immediately before and after hybridization 1 . The base sequence of the target molecules is complementary to the sequence of the probes deposited on the right part of the array; the targets are Cy3 labeled. In hybridization 2 (figure 7), performed afterwards on the same sample, targets are Cy5 labeled and match the probes on the left. Hybridization 1 leads to a stronger negative shift in $U_{S}$ on the right ( 8.3 compared to $1 \mathrm{mV}$ in average), while for hybridization 2 we observe a stronger negative shift on the left ( 8.1 compared to $4.8 \mathrm{mV})$. This is consistent with specific hybridization in both directions.

The specificity of both hybridizations is subsequently confirmed by fluorescence imaging. For this purpose the sample is rinsed with $\mathrm{H}_{2} \mathrm{O}$ and dried. Under excitation at $532 \mathrm{~nm}$ (figure 6, right) we observe 4 times more fluorescence on the right part than on the left part of the array. This way we measure the fluorescence arising from the $\mathrm{Cy} 3$ labeled targets used in the first hybridization (targets matching the probes on the right part of the array). Under excitation at $633 \mathrm{~nm}$ (figure 7) we observe 5 times more fluorescence on the left part than on the right part of the array. This way we measure the fluorescence arising from the Cy5 labeled targets used in hybridization 2 (targets match probes on the left part of the array). Each fluorescence data point corresponds to the average of 3 measurements taken across the active surface of a transistor with a spatial resolution of about $500 \mathrm{~nm}$. The fluorescence measurements are in qualitative agreement with the electronic signals and the signal-to-noise ratios observed with the two different techniques are not very different.

Residual non-specific interactions are expected under our experimental conditions and might explain the signals observed even on the non-matching probes. In particular, here we didn't introduce a blocker step to neutralize the positive charges in the poly(L-lysine) layer that still remain after the deposition of the DNA probes.

The hybridization conditions used in the present experiment (salt, temperature, duration, oligonucleotide length, surface concentrations of probes and hybridized targets) are similar to the conditions used by Fritz et al [10] in ac measurements of oligonculeotide hybridization on capacitive $\mathrm{Si} / \mathrm{SiO}_{2}$ devices and our 
differences in the average shifts $\Delta U_{S}(7.3 \mathrm{mV}$ for hybridization 1 and $3.3 \mathrm{mV}$ for hybridization 2) are close to their result $(3 \mathrm{mV})$.

It is possible to use different salt concentrations for hybridization and detection in order to optimise both the specificity of the sequence recognition (preferentially high salt) and the sensitivity of the field effect detection (preferentially low salt to reduce screening by mobile ions). We have shown experimentally that the differential signals of hybridization can be enhanced significantly (from about $3 \mathrm{mV}$ to $20 \mathrm{mV}$ ) by changing the salt concentration between hybridization and detection [16].

\section{$5 \quad$ Salt dependence of the electronic signals}

Descriptions of the silica surface in aqueous environment [23] and of its modifications induced by biomolecule binding are complex, combining surface chemistry, statistical mechanics and electrostatics. In these systems the concentration of the mobile ions in the electrolyte is an important parameter, controlling the biomolecule interaction with the surface as well as the sensitivity of the electronic detection.

Towards a quantitative understanding of the FET based electronic detection, we performed combined experimental and theoretical studies of the salt dependencies of the electrostatic potentials at the silica/biomolecule/electrolyte interface. This section only gives a glimpse at our study of the salt dependence of a bare silica surface in contact with a $\mathrm{KCl}$ electrolyte. The interested reader is refered to the original references $[12,22]$. The topic is related to the modelisation of $\mathrm{pH}$ measurement by field effect devices [24,25] and to recent publications on the mechanisms of field effect detection of surface bound biomolecules [26-28].

To prepare the experimental study of the salt dependence of the silica/electrolyte interface, we prepare the FET array surface according to Sect. 2.2 without poly(L-lysine) coating. In figure 8, a measurement of the salt dependence of $U_{S E}$ at fixed $I_{D}, U_{S D}$ working point is shown. Starting at $5 \times 10^{-6} \mathrm{M}$, a series of increasing concentration has been obtained by successively adding concentrated $\mathrm{KCl}$ to the electrolyte. The experimental data are compared to two different theoretical descriptions. In the first description (constant charge model, dashed line) we assume an effective interface charge $\sigma_{\text {eff }}$ which does not depend on the salt concentration $[\mathrm{KCl}]$ of the electrolyte. In the second case (salt-dependent charge model, solid line) the dependence of $\sigma_{\text {eff }}$ on $[\mathrm{KCl}]$ is taken into account considering the chemical equilibrium of the ionisable sites at the silica surface. This introduces two fitting parameters, the surface density of ionisable site $N_{S}$ and the $\mathrm{pH}$ value. We find that, although the constant charge model can describe the global trend of the measured salt dependence, the salt-dependent charge model provides a better description of the experimental data. 


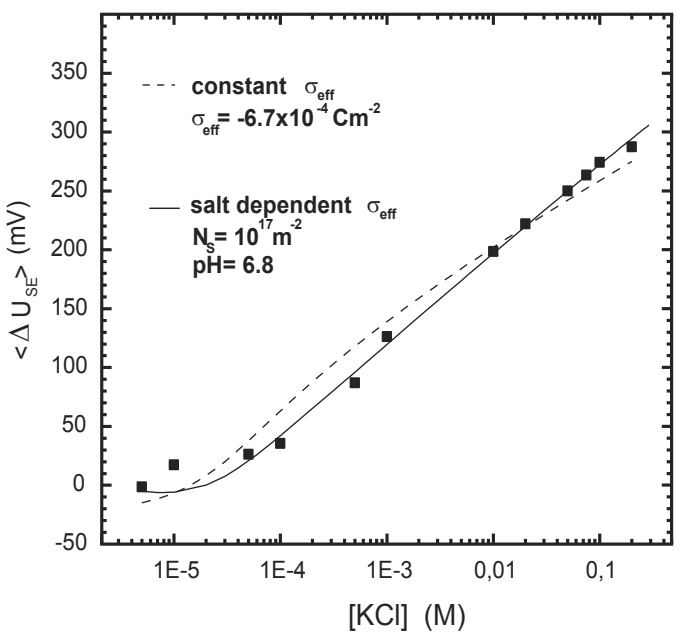

Fig. 8. Average shifts $\Delta U_{S E}$ of an array of 29 transistors as a function of the salt concentration of the buffer solution. Zero voltage shift corresponds to the reference measurement performed with a concentration $[\mathrm{KCl}]$ of $5 \times 10^{-6} \mathrm{M}$. The experimental data (squares) are compared to two different theoretical descriptions as explained in the text.

\section{Concluding remarks}

The electronic detection is sensitive to charged molecules in general. Two different concepts have been used to obtain sequence specific transistor based DNA detection.

In a first approach, point-mutations have been detected in human DNA by allele-specific PCR followed by electronic detection of the double stranded PCR product [15]. The PCR and the electronic detection are separated by a purification that retains PCR product and substrat DNA, but eliminates nucleotides, primers, proteines and salt. As very few substrat DNA is used, the PCR product represents the only species susceptible to induce a significant signal in the electronic detection. Comparative analysis of two different samples on the FET array provides reproducible mutation detection, the overall specificity is simply that of the allele-specific PCR. A combination of FET based DNA detection and sequence specific extension of surface bound oligonucleotides has been reported by Sakata and Miyahara [29].

The second concept consists in obtaining a sequence specific binding on the surface of the FET device by hybridization. Electronic detection of hybridization with probe DNA covalently bound on the surface of FET devices has been reported by several groups $[9,13,14]$. In the work reviewed in Sect. 4 , we immobilize DNA probes on poly(L-lysine) and hybridize oligonucleotide targets at low salt, without preceeding blocker step. The low salt hybridization on a 
charge-compensated surface has initially been introduced to obtain faster hybridization [30]. It has been shown that under these conditions a single base mismatch can be detected in 12 mer oligonucleotides [10], although more standard hybridization protocols use higher concentrations of monovalent salt (50 mM-1M) to improve stringency [31]. For FET array based electronic detection it has been shown experimentally, that the differential signals of hybridization can be enhanced significantly by changing the salt concentration between hybridization and detection [16].

We use devices with multiple transistor structures to detect DNA. Silicon FET arrays can be made with an individual structure size of a few $\mu \mathrm{m}^{2}$. Using silicon CMOS technology, Infineon fabricated 2D sensor arrays with 16384 FET structures and integrated readout electronics on a chip surface of $1 \mathrm{~mm}^{2}$ [17]. As the signals from the individual active FET structures do not decrease with sensor surface and because the dc measurement does not require sophisticated on-chip electronics, this suggests that an electronic dc detection based on a twodimensional transistor array even integrated with on-chip multiplexing would not limit the number of different DNA probes that could be used in parallel. In the field of DNA microarrays, robotic spotting of probe molecules typically gives spot diameters of about $100 \mu \mathrm{m}$, while smaller diameters down to about $5 \mu \mathrm{m}$ are achieved by direct on-surface oligonucleotide synthesis.

The planar $\mathrm{SiO}_{2}$ surface of the FET arrays exhibits sufficiently uniform wetting properties to allow for local spotting and microscopic fluorescence measurements, common in the field of DNA microarrays. In this geometry, integrations with microfluidics approaches and even sophisticated "lab on chip" devices are conceivable and are also potentially interesting in terms of applications.

\section{Acknowledgments}

The contributions of my collaborators C. Gentil, F. Pouthas, D. Côte, G. Philippin and C. Guiducci are gratefully acknowledged. We thank M. Ulbrich, M. Völker, G. Zeck, B. Straub and P. Fromherz for stimulating discussions and help in the preparation of the transistor arrays.

\section{References}

1. Csako G (2006) Clin Chim Acta 363:6-31

2. Wang J, Nielsen P E, Jiang M, Cai X, Fernandes J R, Grant D H, Ozsoz M, Beglieter A, Mowat M (1997) Anal. Chem. 69:5200-5202

3. Steemers F J, Ferguson J A, Walt D R (2000) Nat. Biotech. 98:91-94.

4. McKendry R, Zhang J, Arntz Y, Strunz T, Hegner M, Lang H P, Baller M K, Certa U, Meyer E, Güntherodt H J, Gerber C (2002) Proc. Natl. Acad. Sci. USA 99:9783-9788

5. Bergveld P (1972) IEEE Trans. Biomed. Eng. BME-19:342-351

6. Bergveld P (1996) Sens. Actuators A 56:65-73

7. Kharitonov A B, Wassermann J, Katz E, Willner I (2001) J. Phys. Chem. B 105:4205-4213 
8. Cui Y, Wei Q, Park H, Lieber C M (2001) Science 293:1289-1292

9. Souteyrand E, Cloarec J P, Martin J R, Wilson C, Lawrence I, Mikkelsen S, Lawrence M F (1997) J. Phys. Chem. B 101:2980-2985

10. Fritz J, Cooper E B, Gaudet S, Sorger P K, Manalis S R (2002) Proc. Natl. Acad. Sci. USA 99:14142-14146

11. Hahm J, Lieber C M (2004) Nano Lett. 4:51-54 (2004).

12. Pouthas F, Gentil C, Côte D, Zeck G, Straub B, Bockelmann U (2004) Phys. Rev. E 70:031906

13. Uslu F, Ingebrand S, Mayer D, Böcker-Meffert S, Odenthal M, Offenhäuser A (2004) Biosens. Bioelectron. 19:1723-1731

14. Sakata T, Miyahara Y (2005) ChemBioChem 6:703-710

15. Pouthas F, Gentil C, Côte D, Bockelmann U (2004) Appl. Phys. Lett. 84:15941596

16. Gentil C, Philippin G, Bockelmann U (2007) Phys. Rev. E 75:011926

17. Eversmann B, Jenkner M, Hofmann F, Paulus C, Brederlow R, Holzapfl B, Fromherz P, Merz M, Brenner M, Schreiter M, Gabl R, Plehnert K, Steinhauser M, Eckstein G, Schmitt-Landsiedel D, Thewes R (2003) IEEE J. Solid-St. Circ. 38:2306-2317

18. Kiessling V, Müller B, Fromherz P (2000) Langmuir 16:3517-3521

19. Zeck G, Fromherz P (2001) Proc. Natl. Acad. Sci. USA 98:10457-10462

20. Manning G S (1978) Quart. Rev. Biophys. 11:179-246

21. Pack G R, Wong L, Lamm G (1999) Biopolymers 49:575-590

22. Gentil C, Côte D, Bockelmann U (2006) Phys. Stat. Sol. A 203:3412-3416

23. van Hal R E G, Eijkel J C T, Bergveld P (1996) Adv. Coll. Interf. Sci. 69:31-62

24. Siu W M, Cobbold R S C (1979) IEEE Trans. El. Dev. 26:1805-1815

25. Fung C D, Cheung P W, Ko W H (1986) IEEE Trans. El. Dev. 33:8-18

26. Landheer D, Aers G, McKinnon W R, Deen M J, Ranuarez J C (2005) J. Appl. Phys. 98:044701

27. Neff P A, Wunderlich B K, Lud S Q, Bausch A R (2006) Phys. Stat. Sol. A 203:3417-3427

28. Poghossian A, Cherstvy A, Ingebrandt S, Offenhäuser A, Schoning M J (2005) Sens. Actuators B 111:470-480

29. Sakata T, Miyahara Y (2006) Angew. Chem. Int. Ed. 45:2225-2228

30. Belosludtsev Y, Belosludtsev I, Iverson B, Lemeshko S, Wiese R, Hogan M, Powdrill T (2001) Biochem. Biophys. Res. Commun. 282:1263-1267

31. Sambrook J, Russel D W (2000), Molecular Cloning, A Laboratory Manual. Cold Spring Harbor Laboratory Press, New York 